\title{
Staphylococcus aureus overcomes anaerobe-derived short-chain fatty acid stress via FadX and the CodY regulon
}

Joshua R. Fletcher ${ }^{1}$, Alex R. Villareal ${ }^{1}$, Mitchell Penningroth ${ }^{1}$, Ryan C. Hunter ${ }^{1 *}$

${ }^{1}$ Department of Microbiology \& Immunology, University of Minnesota, 689 23rd Avenue SE, Minneapolis, MN 55455

* To whom correspondence should be addressed:

Ryan C. Hunter

Department of Microbiology \& Immunology

University of Minnesota

689 23rd Ave. SE

Minneapolis, MN 55455

Tel: (612) 625-1402

Email: rchunter@umn.edu

20 Running Title: Mechanisms of $S$. aureus short-chain fatty acid stresses 


\section{Abstract}

Chronic rhinosinusitis (CRS) is characterized by immune dysfunction, mucus hypersecretion, and

24 persistent infection of the paranasal sinuses. While Staphylococcus aureus is a primary CRS pathogen,

25 recent sequence-based surveys have found increased relative abundances of anaerobic bacteria,

26 suggesting that $S$. aureus may experience altered metabolic landscapes in CRS relative to healthy

27 airways. To test this possibility, we characterized the growth kinetics and transcriptome of $S$. aureus in

28 supernatants of the abundant CRS anaerobe Fusobacterium nucleatum. While growth was initially

29 delayed, S. aureus ultimately grew to similar levels as in the control medium. The transcriptome was

30 significantly affected by $F$. nucleatum metabolites, with the agr quorum sensing system notably

31 repressed. Conversely, expression of $\mathrm{fad} X$, encoding a putative propionate coA-transferase, was

32 significantly increased, leading to our hypothesis that short chain fatty acids (SCFAs) produced by $F$.

33 nucleatum could mediate $S$. aureus growth behavior and gene expression. Supplementation with

34 propionate and butyrate, but not acetate, recapitulated delayed growth phenotypes observed in $F$.

35 nucleatum supernatants. A fadX mutant was found to be more sensitive than wild type to propionate,

36 suggesting a role for FadX in the S. aureus SCFA stress response. Interestingly, spontaneous resistance

37 to butyrate, but not propionate, was frequently observed. Whole genome sequencing and targeted

38 mutagenesis identified $\operatorname{cod} Y$ mutants as resistant to butyrate inhibition. Together, these data show that $S$.

39 aureus physiology is dependent on its co-colonizing microbiota and metabolites they exchange, and

40 indicate that propionate and butyrate may act on different targets in S. aureus to suppress its growth. 


\section{Importance}

42 S. aureus is an important CRS pathogen, yet is found in the upper airways of $30-50 \%$ of people without

43 complications. The presence of strict and facultative anaerobic bacteria in CRS sinuses has recently

44 spurred research into bacterial interactions and how they influence $S$. aureus physiology and

45 pathogenesis. We show here that propionate and butyrate produced by one such CRS anaerobe, $F$.

46 nucleatum, alter the growth and gene expression of $S$. aureus. We show that fadX is important for $S$.

47 aureus to resist propionate stress, and that the CodY regulon mediates growth in inhibitory concentrations

48 of butyrate. This work highlights the possible complexity of $S$. aureus-anaerobe interactions, and

49 implicates membrane stress as a possible mechanism influencing $S$. aureus behavior in CRS sinuses. 


\section{INTRODUCTION}

Chronic rhinosinusitis (CRS) is an inflammatory condition of the sinuses that is broadly characterized by facial pain, mucus hypersecretion and accumulation, immune dysfunction, pathogen colonization, and persistent polymicrobial infection ${ }^{1-6}$. Although CRS affects up to $15 \%$ of the population and represents a substantial economic burden, its complexity has slowed development of new treatments and therapeutic strategies ${ }^{7}$. CRS patients are frequently prescribed antibiotics, yet many do not respond and require functional endoscopic sinus surgery (FESS) to remove accumulated mucus and inflamed mucosa that prevents proper sinus drainage ${ }^{5}$. Given the urgent threat of antimicrobial resistance among CRS microbiota, there is a critical need to better understand microbial community dynamics in the upper airways and how they may contribute to disease ${ }^{8}$.

Staphylococcus aureus is a frequently isolated CRS pathogen and is aggressively targeted by

61 antibiotic therapy, yet, this bacterium is also prevalent and abundant in the upper airways of 62 asymptomatic healthy individuals ${ }^{9,10}$. This seeming paradox suggests that colonization by $S$. aureus is not sufficient to drive disease, but rather that there may be important environmental cues in the upper airways that shift the lifestyle of $S$. aureus towards commensalism or pathogenesis. Indeed, in a genomewide association study of $S$. aureus isolated from 28 CRS patients, few $S$. aureus genetic signatures were associated with CRS subtypes, suggesting that $S$. aureus pathogenesis in CRS is unlikely due to selection for increased production of a particular toxin ${ }^{11}$. a small number of etiologic bacterial species toward a polymicrobial basis of disease $e^{4,5,12}$. However, the role of the greater CRS microbiome in disease pathophysiology remains poorly understood. To address

71 this knowledge gap, we recently surveyed 16S rRNA gene sequences in FESS-derived mucus from a 72 cohort of CRS patients and found increased relative abundances of numerous anaerobic bacterial taxa, 73 including many known to degrade mucin glycoproteins ${ }^{6}$. CRS bacterial communities enriched on mucins

74 as a sole carbon source converged on similar profiles, typically dominated by a combination of 75 Streptococcus, Prevotella, Fusobacterium, and Veillonella. Interestingly, S. aureus had a variety of 76 growth phenotypes and gene expression patterns when cultured in supernatants from these enrichment 
77 communities, indicating that nutrient usage and metabolite release by co-colonizing microbiota can

78 profoundly affect $S$. aureus physiology ${ }^{6}$. Enrichment supernatants that best supported S. aureus growth

79 had low levels of short-chain fatty acids (acetate, propionate, butyrate; SCFAs) and undetectable levels

80 of Fusobacterium, members of which are known for producing SCFAs as amino acid fermentation

81 byproducts ${ }^{13,14}$. However, neither growth promotion nor inhibition could be ascribed to any one taxon or

82 metabolite within these communities.

83 In this study, we extend our previous work by demonstrating that $F$. nucleatum metabolites

84 impede $S$. aureus growth and repress transcription of the accessory gene regulator (agr) quorum sensing

85 system while inducing a putative fatty acid degradation operon (fadXEDBA). We confirm that the SCFAs

86 propionate and butyrate are sufficient to impair S. aureus growth and alter gene expression, while acetate

87 had relatively little effect. We show that growth of a $\Delta$ fadX mutant is significantly attenuated in the

88 presence of propionate only, despite differing from butyrate by only one carbon. Spontaneous resistance

89 to growth inhibition by butyrate arose frequently, while we failed to obtain propionate resistant mutants.

90 Genome sequencing of butyrate resistant mutants identified premature stop codons and in-frame

91 deletions in the gene encoding the nutrient-responsive global regulator $\operatorname{cod} Y$, indicating a connection

92 between de-repression of the CodY regulon through nutrient limitation and SCFA resistance. These data

93 suggest that certain anaerobes may influence CRS community structure by limiting $S$. aureus growth via

94 propionate and butyrate production. In addition, they implicate the CodY regulon as a mechanism

95 allowing $S$. aureus persistence in otherwise inhospitable anaerobic bacterial communities of the upper

96 airways.

97

98 MATERIALS AND METHODS

99 Bacterial strains and growth conditions. Bacterial strains used throughout this study are shown in 100 Table S1. Plasmids and primers used for mutagenesis and complementation can be found in Table S2 101 and S3, respectively. Staphylococcus aureus strains USA300 LAC, JE2 and fadX::tn transposon mutant 102 (obtained from the Nebraska Transposon Mutant Library) were routinely cultured aerobically at $37^{\circ} \mathrm{C}$ on 103 LB agar (IBI Scientific IB49020) or with shaking at 220 rpm in LB broth, both supplemented as needed 
with $10 \mu \mathrm{g} / \mathrm{mL}$ chloramphenicol $(\mathrm{Cm} \text {, Teknova } \mathrm{C} 0325)^{6,15}$. S. aureus was also grown in cell free supernatants (CFS) of Fusobacterium nucleatum ATCC 25586 that had been cultured anaerobically for $48 \mathrm{~h}$ in BBL Brucella Broth (BD 2011088) supplemented with $250 \mu \mathrm{g} / \mathrm{mL}$ and $50 \mu \mathrm{g} / \mathrm{mL}$ of hemin and vitamin K (Hardy Diagnostics Z237), respectively. To test the effects of specific SCFAs on S. aureus growth and gene expression, the sodium salts of acetate (Fisher Scientific S209), propionate (Sigma P1880), or butyrate (Sigma 303410) were added at various concentrations to LB then passed through a

111 (ThermoFisher Scientific C404010) was used for cloning the fadX mutagenesis plasmid while E. coli DC10B was used for plasmid passaging to prevent cytosine methylation to facilitate easier transfer to $S$. aureus. E. coli strains were routinely grown on and in LB with $20 \mu \mathrm{g} / \mathrm{mL} \mathrm{Cm}$ or $100 \mu \mathrm{g} / \mathrm{mL}$ ampicillin (Amresco 0339) as needed.

Growth curves. Overnight cultures of wild type S. aureus and various mutants were diluted 1:100 in sterile PBS, then $5 \mu \mathrm{L}$ was added to $195 \mu \mathrm{L}$ of growth medium per well in a 96 well microtitre dish. Plates were incubated at $37^{\circ} \mathrm{C}$ for $24 \mathrm{~h}$ in a BioTek Synergy $\mathrm{H} 1$ microplate reader for $24 \mathrm{~h}$, with five seconds of orbital shaking performed prior to hourly $\mathrm{OD}_{600}$ readings.

Biofilm quantification. The crystal violet staining of biofilm material was performed according to Merritt 120 et $\mathrm{al}^{16}$. Briefly, overnight $S$. aureus LB cultures were centrifuged at 5,000 rpm for 5 minutes and washed 121 once with sterile PBS. They were sub-cultured 1:100 into fresh media in a 96 well microtiter plate and 122 incubated statically at $37^{\circ} \mathrm{C}$ for $48 \mathrm{~h}$, after which time the $\mathrm{OD}_{600}$ values were recorded in a BioTek Synergy $123 \mathrm{H} 1$ microplate reader. Plates were then inverted to remove the cultures, washed three times in water, 124 and allowed to dry. The wells were stained with $0.1 \% \mathrm{w} / \mathrm{v}$ crystal violet for 15 minutes at room 125 temperature. The crystal violet was removed, and the plates were washed a further three times in water 126 and allowed to dry. The dye was solubilized with $30 \%$ acetic acid for 15 minutes and then the absorbance 127 at $560 \mathrm{~nm}$ was recorded. The $\mathrm{OD}_{560}$ was normalized to the $\mathrm{OD}_{600}$ for each well to generate the final 128 values. 
RNA extraction. For S. aureus growth in anaerobe cell-free supernatants and in LB with or without SCFA supplementation, $2 \mathrm{~mL}$ of growth medium was inoculated 1:100 with $S$. aureus overnight LB cultures and grown at $37^{\circ} \mathrm{C}$ with shaking at $220 \mathrm{rpm}$. Growth of each culture was monitored until they reached an $\mathrm{OD}_{600}$ of $\sim 0.2$ to 0.3 , after which they were centrifuged for $1 \mathrm{~min}$ at $14,000 \mathrm{rpm}$. Supernatants were discarded, and pellets suspended in $50 \mu \mathrm{L}$ of fresh LB supplemented with $20 \mu \mathrm{g} / \mathrm{mL}$ of lysostaphin (Sigma-Aldrich L7386). These were then incubated in a $37^{\circ} \mathrm{C}$ water bath for $15-20$ min or until the suspension cleared (no longer than $30 \mathrm{~min}$ ). One $\mathrm{mL}$ of TRIzol Reagent (ThermoFisher 15596018) was added to the lysate, pipetted gently until mixed, and incubated at room temperature (RT) for 5 min. 200 $\mu \mathrm{L}$ of chloroform (VWR 0757) was added per tube and samples were vigorously shaken for $15 \mathrm{~s}$, then incubated at RT for $5 \mathrm{~min}$. Phase separation was performed by centrifugation at 12,000 rpm for $15 \mathrm{~min}$ at $4^{\circ} \mathrm{C}$. $\sim 500 \mu \mathrm{L}$ of the aqueous phase was removed and added to $500 \mu \mathrm{L}$ of $95 \%$ ethanol (Decon Laboratories, Inc. UN1170), vortexed for $5 \mathrm{~s}$ and incubated at RT for $5 \mathrm{~min}$. RNA was then isolated using the Zymo RNA Clean \& Concentrator-5 kit according to the manufacturer's instructions, including an oncolumn DNase I treatment.

NanoString analysis of $\boldsymbol{S}$. aureus gene expression. A custom NanoString probe set (Table S4) was designed to target transcripts for several key S. aureus virulence factors, metabolic genes, and global regulators. The probe set also included six housekeeping genes for normalization. DNase I-treated RNA from S. aureus grown in triplicate to $\mathrm{OD}_{600} \sim 0.2$ to 0.3 in control medium (Brucella Broth, $\mathrm{BB}$ ) or $48 \mathrm{~h}$ cellfree supernatants from F. nucleatum (Fn CFS) was submitted to the University of Minnesota Genomics Center (UMGC) for hybridization to the custom probe set. Raw data were imported into the nSolver Advanced Analysis software package for normalization and differential gene expression analysis using default settings. Transcripts were considered differentially expressed if their levels changed by two-fold and the Benjamini-Hochberg adjusted p-value was less than 0.05 . The heatmap was constructed using the pheatmap package (v.1.0.12) in $\mathrm{R}(\mathrm{v} .4 .1 .0)^{17}$.

Reverse transcription and quantitative real-time PCR. $1.5 \mu \mathrm{g}$ of RNA was reverse transcribed using M-MuLV Reverse Transcriptase (NEB M0253L) following the manufacturer's protocol. cDNA was diluted 
1:15 in sterile water prior to use in qRT-PCR using SsoAdvanced Universal SYBR Green Supermix (Bio Rad 1725271). PCR products for each gene being assayed (see Table S3 for primer sequences) were used to construct standard curves for quantification. To determine relative copy number, transcript levels were normalized to the housekeeping gene gmk (guanylate kinase), which was confirmed to be consistent across growth conditions.

Construction of a S. aureus $\Delta \mathrm{fadX}$ deletion mutant. $\sim 500$ bp sequences flanking the fadX gene (SAUSA300_0229) were amplified by PCR using Q5 DNA Polymerase (NEB M0491L). For cloning purposes, the upstream amplicon contained a $5^{\prime} \mathrm{Kpnl}$ restriction site and the downstream amplicon contained a 3' Sacl site, while the internal ends contained complementary overhangs to facilitate overlap extension PCR to fuse the fragments together. The final product was $\mathrm{a} \sim 1 \mathrm{~kb}$ fragment encoding the first 11 codons and the stop codon of fadX. The amplicon was digested with Kpnl-HF (NEB R3142S) and Sacl-HF (NEB R3156S) and cloned into the temperature sensitive, counter-selectable mutagenesis plasmid pIMAY with T4 DNA ligase $(\mathrm{NEB} M 0202)^{18}$. The $\Delta$ fadX plasmid was transformed into $E$. coli $\mathrm{DC10B}$, then electroporated $(2900 \mathrm{~V}, 25 \mu \mathrm{F}, 100 \Omega)$ in a $2 \mathrm{~mm}$ cuvette into $\mathrm{S}$. aureus. The culture was grown at the plasmid replication permissive temperature of $28^{\circ} \mathrm{C}$ with shaking for $4 \mathrm{~h}$, after which time it was plated onto LB $+10 \mu \mathrm{g} / \mathrm{mL} \mathrm{Cm}$ and incubated on the benchtop for $48 \mathrm{~h}$. The single colony that was obtained was streaked onto two LB $+\mathrm{Cm}$ plates. One was incubated on the benchtop for $48 \mathrm{~h}$ to generate a freezer stock and the other was incubated at $37^{\circ} \mathrm{C} . \mathrm{Cm}$-resistant colonies were screened via PCR for chromosomal integration of the plasmid. Positive colonies were grown in LB in the absence of selection at $37^{\circ} \mathrm{C}$ for $24 \mathrm{~h}$, subcultured $1: 1000$ into fresh LB for another $24 \mathrm{~h}$, then plated onto $\mathrm{LB}+1 \mu \mathrm{g} / \mathrm{mL}$ anhydrotetracycline hydrochloride (Sigma Aldrich 37919) for counter-selection via induction of the TetRregulated $\sec Y$ antisense RNA and incubated overnight at $37^{\circ} \mathrm{C}$. Resultant colonies were patched onto 177 fresh counter-selection agar and LB $+\mathrm{Cm}$ to screen for loss of the plasmid. Cm-sensitive clones were screened by PCR for loss of fadX coding sequence and four were confirmed as mutants by Sanger sequencing. 
butyrate, and propionate in cell free supernatants. Organic acids of interest were purified from complex media components through a modified liquid-liquid extraction method ${ }^{19}$. To account for analyte loss during extraction, $100 \mu \mathrm{L}$ of $0.2 \mathrm{M}$ succinate was added as an internal standard to $2 \mathrm{mLs}$ of each sample $(9.5 \mathrm{mM} \text { final concentration })^{19}$. After equilibration at room temperature for $5 \mathrm{~min}, 200 \mu \mathrm{L}$ of $12 \mathrm{~N} \mathrm{HCl}$ was added, and samples were vortexed for 15 seconds. $10 \mathrm{~mL}$ of diethyl ether was then added to each sample before gently rolling them for a total of $30 \mathrm{~min}$. After centrifugation for $5 \mathrm{~min}$ at 4000rpm, supernatants were transferred to a new extraction tube and $1 \mathrm{~mL}$ of $1 \mathrm{M} \mathrm{NaOH}$ was added before gently rolling for another $30 \mathrm{~min}$. The resulting aqueous phase was extracted and transferred to an autosampler vial followed by addition of $100 \mu \mathrm{L}$ of $12 \mathrm{~N} \mathrm{HCL}$ before vortexing and storage at $4^{\circ} \mathrm{C}$ until analysis. with a reversed-phase Acclaim Organic Acid (OA) Column ( $5 \mu \mathrm{m}, 120 \mathrm{~A}, 4.0 \times 250 \mathrm{~mm}) .8 \mu \mathrm{L}$ of each sample was injected and separation was achieved using a 32-minute isocratic instrument method (1.0 $\mathrm{mL} / \mathrm{min}, 30^{\circ} \mathrm{C}$ ) employing $\mathrm{Na}_{2} \mathrm{SO} 4(100 \mathrm{mM}, \mathrm{pH} 2.6)$ as the mobile phase. The column was allowed to equilibrate for $8 \mathrm{~min}$ prior to sample injection. Absorbance was monitored at $210 \mathrm{~nm}$ to identify compounds with a carboxylic acid functional group. Chromatograms were processed using Dionex Chromeleon 7 (Thermo Fisher) Chromatography Data System. Cobra Wizard was used to reproducibly identify and gate peaks of interest.

Isolation and genome sequencing of butyrate resistant mutants. The $\Delta$ fadX mutant was grown for

24 hours in LB, serially diluted, plated onto LB supplemented with $200 \mathrm{mM}$ sodium butyrate, and incubated overnight at $37^{\circ} \mathrm{C}$. Four large colonies were re-streaked onto the same media to confirm their ability to grow in the presence of butyrate. Genomic DNA was then isolated from our S. aureus USA300 LAC isolate and butyrate-resistant mutants using the PowerSoil Pro kit (Qiagen 47014). Overnight cultures were pelleted and suspended in $200 \mu \mathrm{L}$ of an enzymatic lysis buffer $(20 \mathrm{mM}$ Tris- $\mathrm{HCl}, 2 \mathrm{mM}$ EDTA, $1.2 \%$ (vol/vol) Triton $\mathrm{X}-100$, and $20 \mu \mathrm{g} / \mathrm{mL}$ lysostaphin) for $30 \mathrm{~min}$ at $37^{\circ} \mathrm{C}$. Lysates were transferred to Power Bead tubes and the manufacturer's protocol was followed with no further alterations. Genomic DNA was processed and sequenced on the Illumina MiSeq platform at the Microbial Genome 
208 Sequencing Center (MiGS, Pittsburgh, PA). Raw paired-end fastq files were imported into Geneious

209 v.2022.0.1 and trimmed for quality using BBDuk with the following settings: Set ORDERED to true, $k=27$, $210 \operatorname{mink}=6$, maskMiddle=true, hamming distance=1, right-ktrimming using 1 reference, quality-trimming both 211 ends to Q30. Trimmed reads for our USA300 isolate were mapped to the S. aureus subsp. aureus 212 USA300_FPR3757 reference genome (CP000255) using the Geneious Mapper on medium-low 213 sensitivity with a minimum mapping quality of 20 and only mapping reads whose pair mapped 214 appropriately nearby. The assembled USA300 genome was then used as the reference against which 215 reads from the butyrate resistant mutants were mapped using the same quality trimming and mapping 216 strategy detailed above. Putative SNPs and indels were detected using the "Find Variations/SNPS..."

217 function within Geneious, requiring occurrence of the variation in $>90 \%$ of reads, with a minimum of 10 218 reads.

219 Data availability. Raw sequences were deposited at NCBI's Sequence Read Archive (SRA) with the 220 BioProject ID PRJNA798706.

\section{RESULTS}

223 S. aureus growth is impaired by $\boldsymbol{F}$. nucleatum. In a previous study of upper airway (CRS) microbiota, 224 we found that in patients $(n=27)$ with detectable Fusobacterium, relative abundances of Staphylococcus 225 were minimal or below the level of detection (Fig. 1A) ${ }^{6}$. When S. aureus was grown in supernatants 226 derived from anaerobic enrichment cultures of CRS sinus mucus, it exhibited slower growth in those that 227 had Fusobacterium as a core constituent genus of the enrichment community. These supernatants also 228 contained higher levels of the short-chain fatty acids (SCFAs) propionate and butyrate ${ }^{6}$. Given these 229 data, we hypothesized that Fusobacterium spp. exert an antagonistic effect on $S$. aureus through the 230 production of SCFAs. To test this, we grew S. aureus USA300 LAC in filtered cell-free supernatants 231 (CFS) from F. nucleatum ATCC 25586 grown for $48 \mathrm{~h}$ in Brucella Broth (BB) supplemented with hemin 232 and vitamin K (Fig. 1B). S. aureus grew in Fn CFS, albeit slower than in the control medium (BB) with an 233 increased lag phase. However, both cultures reached approximately similar OD $_{600}$ values by 24 h, 234 indicating that $S$. aureus was able to obtain sufficient nutrients over the course of the experiment. We 
reasoned that the extended lag phase was likely due to F. nucleatum-mediated depletion of an easily metabolizable nutrient source and/or the presence of an inhibitory metabolite(s) that $S$. aureus was able to adapt to over time. We tested the latter by measuring acetate, propionate, and butyrate content of the CFS before and after S. aureus growth (Fig. 1C). All three SCFAs were detected in F. nucleatum CFS ( $\sim 5 \mathrm{mM}$ acetate, $\sim 5 \mathrm{mM}$ propionate, $\sim 15 \mathrm{mM}$ butyrate). After overnight growth $(\sim 16 \mathrm{~h})$ in $F$. nucleatum supernatants, S. aureus cultures had increased acetate levels, while propionate and butyrate remained the same as in CFS alone, indicating that $S$. aureus does not actively metabolize the latter two SCFAs under these conditions. We interpret this to mean that $S$. aureus adapts to SCFAs by modifying its physiology rather than directly detoxifying them via degradation. The increased acetate levels are likely due to $S$. aureus utilization of glucose remaining in Fn CFS, as F. nucleatum preferentially ferments amino acids ${ }^{20,21}$. Given that $S$. aureus growth is similarly impaired when BB is supplemented with the sodium salts of acetate $(5 \mathrm{mM})$, propionate $(5 \mathrm{mM})$, and butyrate $(15 \mathrm{mM})(\mathrm{Fig} .1 \mathrm{~B})$, these data suggest that SCFAs may be key factors driving bacterial interactions in the CRS sinus environment, providing a mechanism by which Fusobacterium and other anaerobes may restrict S. aureus growth in vivo.
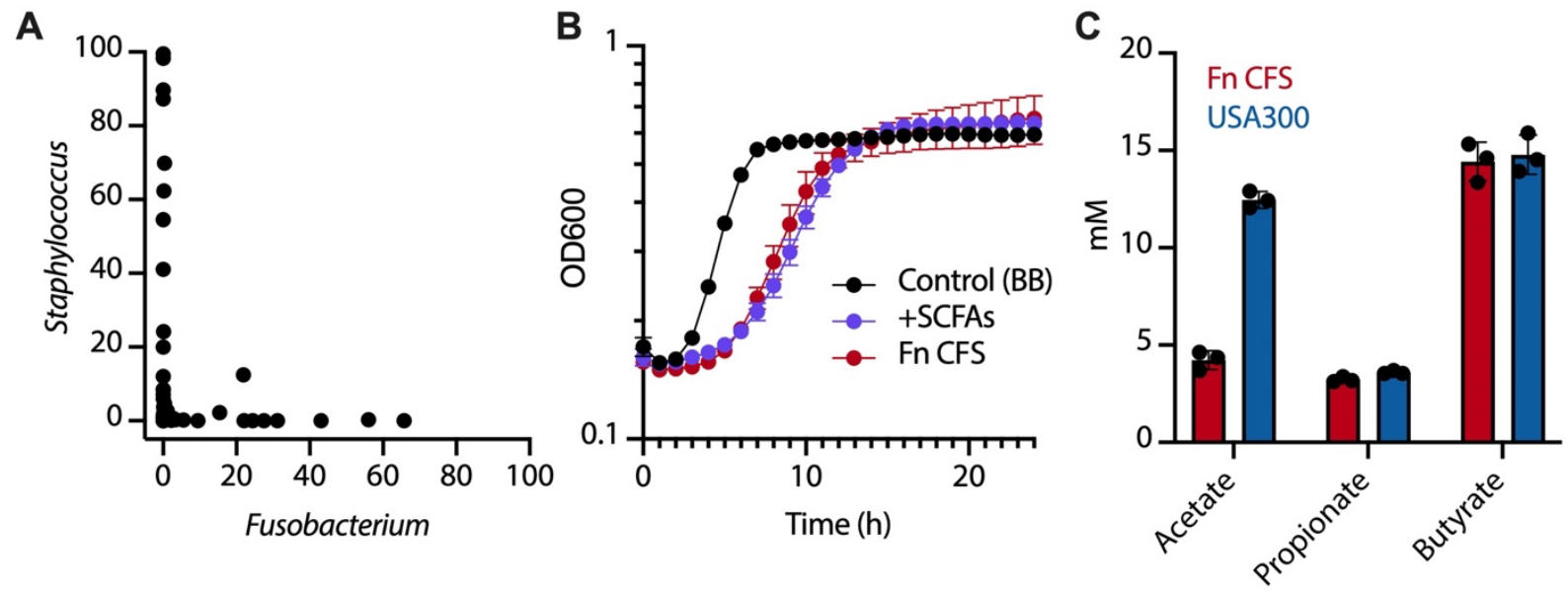

Figure 1. S. aureus growth is impaired in F. nucleatum supernatants. A) Relative abundances of Fusobacterium and Staphylococcus in sinus mucus from patients with chronic rhinosinusitis are inversely correlated (Lucas, et al 2021). B) Representative growth curve of $S$. aureus USA300 in brucella broth (BB, control), BB supplemented with $5 \mathrm{mM}$ acetate, $5 \mathrm{mM}$ propionate, and $15 \mathrm{mM}$ butyrate, and cell-free supernatants from $F$. nucleatum (Fn CFS). C) Production of SCFAs by $F$. nucleatum after 48h (Fn CFS) and their levels after S. aureus (USA300) growth in Fn CFS. 
F. nucleatum metabolites significantly alter S. aureus gene expression. We next determined how S. aureus modified its transcriptome in F. nucleatum CFS. To do so, we performed a targeted analysis using a custom NanoString code set (Table S4) that included 34 genes encoding several known virulence factors, key metabolic genes, and master regulators of gene expression (Fig. 2A). Of these genes, we detected thirteen differentially expressed transcripts ( $\geq 2$-fold change in expression and adjusted pvalue<0.05); expression of $\operatorname{fad} X, \operatorname{cid} A, i c a B$, and gltB increased while nanA, alsS, $\operatorname{Irg} A$, narG, agrA, hla, $h / d$, saeR, and Idh1 decreased in S. aureus grown on CFS relative to BB alone (Fig. 2B). A number of other transcripts (aur, fib, pgi, codY, opp3b, and arIR) were statistically significant, but exhibited less than two-fold change in expression (Table S4). Decreased signaling through the quorum-sensing response regulator agrA in Fn CFS results in lower expression of the hla and hld genes, encoding alpha and delta hemolysins ${ }^{22}$. Neuraminate lyase, encoded by nanA, is induced by the presence of sialic acids and exhibited lower expression in Fn CFS, indicating that $F$. nucleatum likely utilized sialic acids present in Brucella Broth as a nutrient source ${ }^{23,24}$. Expression of the nutrient-sensing transcriptional regulator $\operatorname{cod} Y$ was reduced in Fn CFS by nearly $50 \%$ compared to the control medium, likely explaining the increase in the glutamate synthase subunit gene $g / t B^{25}$. We selected three genes ( $f a d X$, agr $A$, and nanA) for validation via quantitative reverse-transcription PCR and show that they were highly correlated with the

271 NanoString results (Fig. S1). That nearly half of the transcripts in the NanoString probe set are 272 differentially regulated in Fn CFS, including a number of major transcriptional regulators important for 273 integrating metabolic cues and virulence gene expression, highlights the global nature of alterations to 274 the S. aureus transcriptome. These data show that S. aureus physiology can be significantly influenced 275 by the metabolic activity of a single anaerobic species and underscore the possible complexity of bacterial behaviors and interactions within a diverse CRS community. 
A

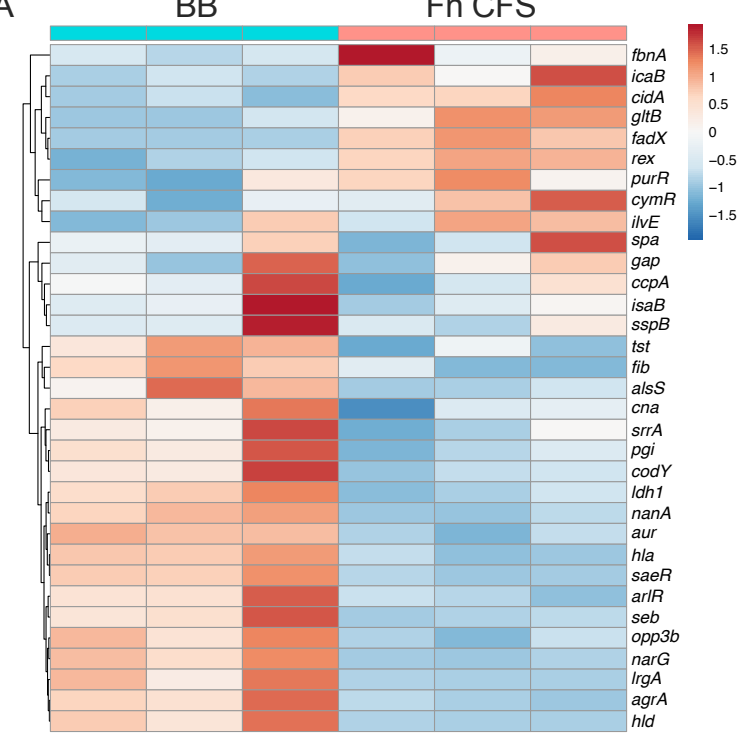

277

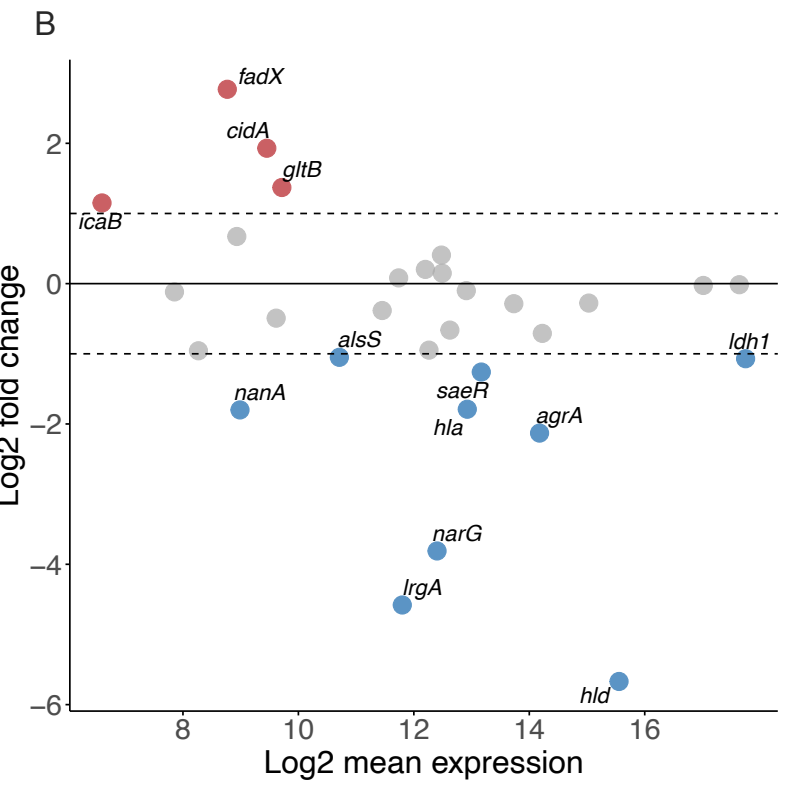

Figure. 2. F. nucleatum metabolites significantly impact the $S$. aureus transcriptome. A) Heatmap depicting log10-transformed $S$. aureus gene expression in control media (BB) and $F$. nucleatum supernatant (CFS) as detected by NanoString. Genes were clustered with unsupervised hierarchical clustering. B) MA plot representation of $S$. aureus gene expression in Fn CFS relative to the control medium. Genes were considered significant if they had a log2 fold change $\geq 1$ and a Benjamini-Hochberg adjusted $p$-value $<0.05$.

SCFAs significantly alter S. aureus gene expression. SCFAs, especially propionate and butyrate, have been reported to impair $S$. aureus growth and attenuate murine skin infections ${ }^{26}$. We therefore sought to determine if individual SCFAs were sufficient to drive some of the S. aureus gene expression patterns observed after growth in Fn CFS. To measure the effects of each SCFA on the agr quorum sensing system, we grew S. aureus carrying pAH1 (encoding Pagr-mCherry) in LB or LB supplemented with acetate, propionate, or butyrate for $24 \mathrm{~h}$ and measured fluorescence intensity normalized to culture density (Fig. 3A). All three SCFAs led to decreased fluorescence, with propionate $(p=0.0035)$ and butyrate $(p<0.0001)$ significantly inhibiting reporter activity, while acetate $(p=0.203)$ had the least effect. Given these observations, CRS bacterial communities dominated by Fusobacterium and other taxa that produce propionate and butyrate would not only be predicted to impede the growth of $S$. aureus, but also minimize the production of agr-regulated virulence factors.

Given the reduction in agr quorum sensing activity and thus lack of repression of proteins involved in surface attachment, we hypothesized that SCFAs may be a pro-biofilm signal to $S$. aureus ${ }^{27}$. We 
performed qRT-PCR on $S$. aureus grown to $\mathrm{OD}_{600} \sim 0.2-0.3$ in LB or LB supplemented with $100 \mathrm{mM}$ of each individual SCFA to determine if biofilm-associated transcripts identified as differentially regulated in Fn CFS were affected. We found that expression of cidA, encoding a holin-like protein involved in programmed cell death and extracellular DNA release during biofilm formation, was approximately tenfold higher $(p<0.0001)$ in the presence of propionate, but was relatively unaffected by acetate or butyrate (Fig. 3B $)^{28}$. Conversely, there was no effect of SCFAs on the expression of $\operatorname{Irg} A$, which encodes a putative anti-holin that is antagonistic to $\mathrm{CidA}^{28}$. This suggests that decreased $\operatorname{IrgA}$ expression detected in Fn CFS is likely indepdendent of the SCFAs tested here (Fig. 3C). Despite increased cidA expression, SCFA supplementation of LB had marginal effects on biofilm production, with acetate and propionate leading inducing modest but insignificant increases relative to LB alone, and butyrate having no detectable effect (Fig. 3D). The lack of downregulation of IrgA under these conditions suggests that sufficient LrgA protein may be available to offset any increased CidA activity. Alternatively, other environmental cues may be
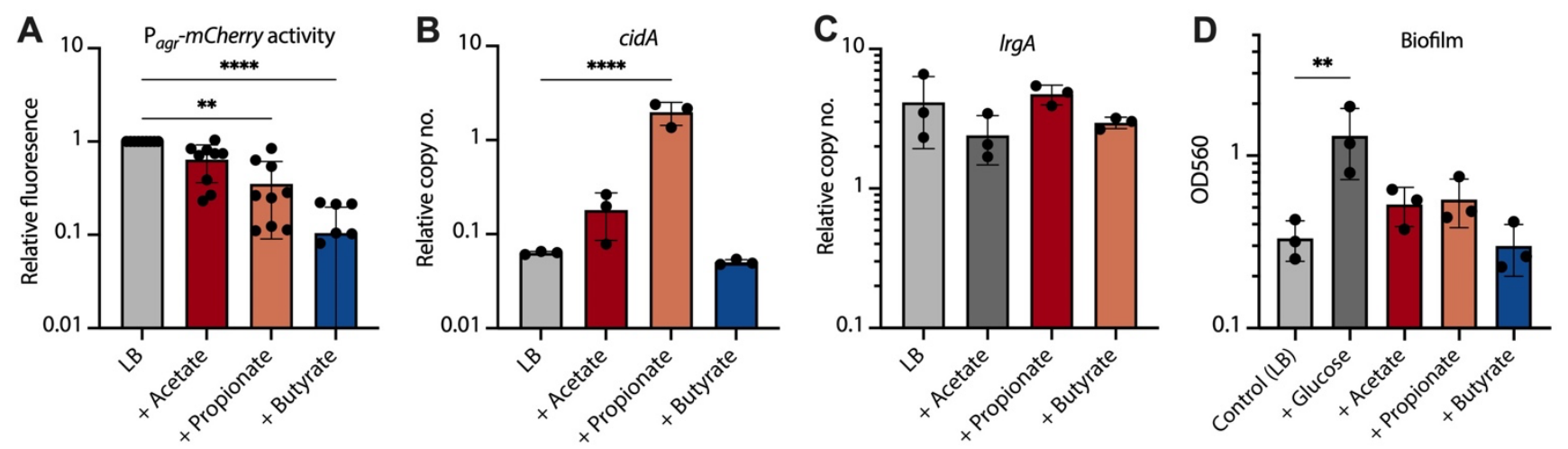

Fig. 3. Propionate and butyrate repress the $S$. aureus agr system but fail to induce biofilm. A) $S$. aureus carrying pAH1 (Pagr-mCherry) was grown for 24 hours in LB supplemented with $100 \mathrm{mM}$ of sodium acetate, propionate, or butyrate ( $n=3$ biological replicates with $n=3$ cultures per replicate). Fluorescence was measured and normalized to culture density for each replicate, then normalized to the LB controls. B, C) Expression of cidA and $\operatorname{IrgA}$ from $S$. aureus in LB supplemented with SCFAs $(\mathrm{n}=3)$. Copy number was determined via standard curve and normalized to the gmk housekeeping gene. D) Crystal violet assay quantifying biofilm formation in LB, LB supplemented with glucose (positive control for increased biofilm formation), or LB supplemented with $100 \mathrm{mM}$ of each SCFA. 
The fadX gene mediates propionate resistance. The most highly induced transcript in S. aureus grown

324 in Fn CFS was fadX, encoding a putative propionate CoA-transferase, the first in a five gene operon 325 predicted to be involved in fatty acid degradation. Given their annotation, we hypothesized that the fad 326 operon may encode a component of the S. aureus SCFA stress response. The fad genes were induced 327 after growth in Fn CFS and in the presence of propionate and butyrate; their status as an operon was 328 confirmed by obtaining amplicons from cDNA using PCR primer sets that spanned each intergenic region 329 (Fig. 4, Fig. S2). We tested a fadX transposon mutant (obtained from the Nebraska Transposon Mutant 330 Library) and its parental strain (JE2) for the ability to grow in $100 \mathrm{mM}$ propionate, and found that fadX::tn 331 had a significant growth defect in propionate relative to the wild type (Fig. S3). The mutant grew as well 332 as the parent strain in LB alone, indicating that growth inhibition was specific to propionate in the medium. 333 We then performed dose-response growth curves in six concentrations of sodium propionate, ranging 334 from $100 \mathrm{mM}$ to $3.125 \mathrm{mM}$ in two-fold reductions, and found clear growth differences between JE2 and 335 fadX::tn, with the mutant having a defect in media with as low as $12.5 \mathrm{mM}$ (Fig. S4A). To determine if the transposon insertion in the fadX::tn mutant disrupted the entire fad operon, 337 we performed qRT-PCR and confirmed that the three genes downstream of fadX had considerably 338 reduced expression in LB (Fig. S4B). We therefore constructed a $\Delta$ fadX deletion mutant in the USA300 339 LAC background and tested its growth in LB supplemented with each SCFA (Fig. 5). Relative to wild 340 type, there was no growth defect in acetate, however there was modest inhibition of the mutant in 341 propionate, with growth curves diverging after approximately 8-10 hours and remaining consistent 342 through the end of the experiments. Neither strain grew well in butyrate, though sporadic growth was 343 detected after $\sim 15 \mathrm{~h}$, irrespective of genotype and only in butyrate. Together, these data implicate FadX 344 in ameliorating or resisting propionate stress, though the mechanism remains unclear. Further, the 345 occasional growth of either strain at later time points in butyrate, but not propionate, provides indirect 346 evidence that these SCFAs may have different mechanisms of S. aureus growth inhibition. 
A

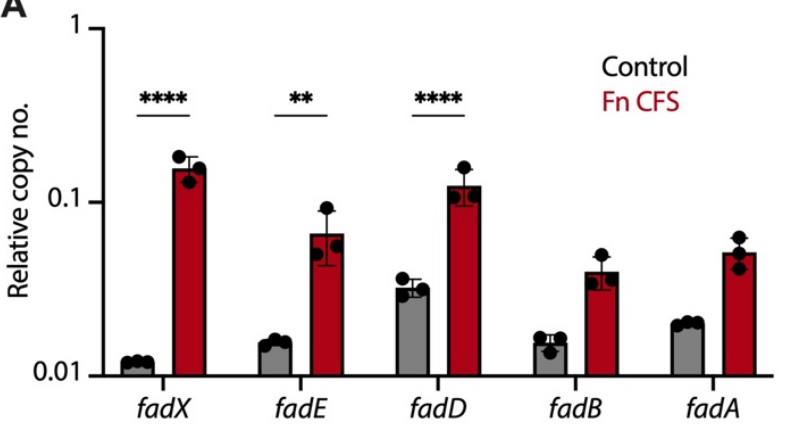

B

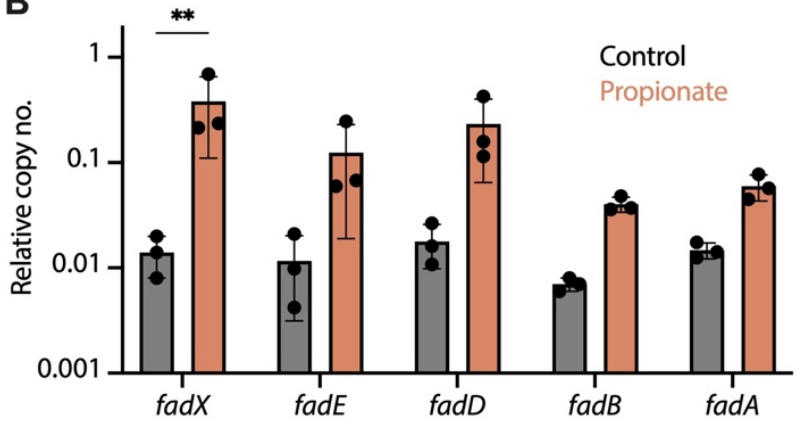

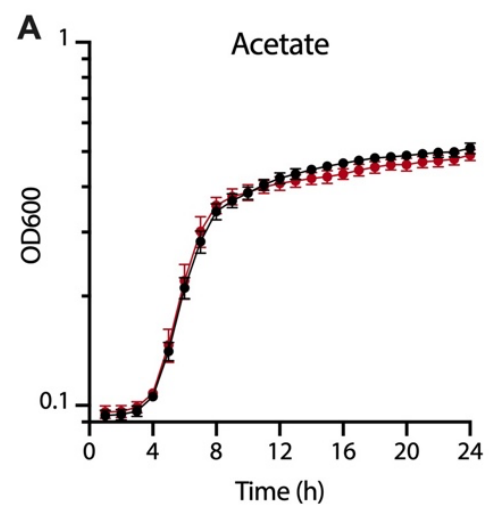
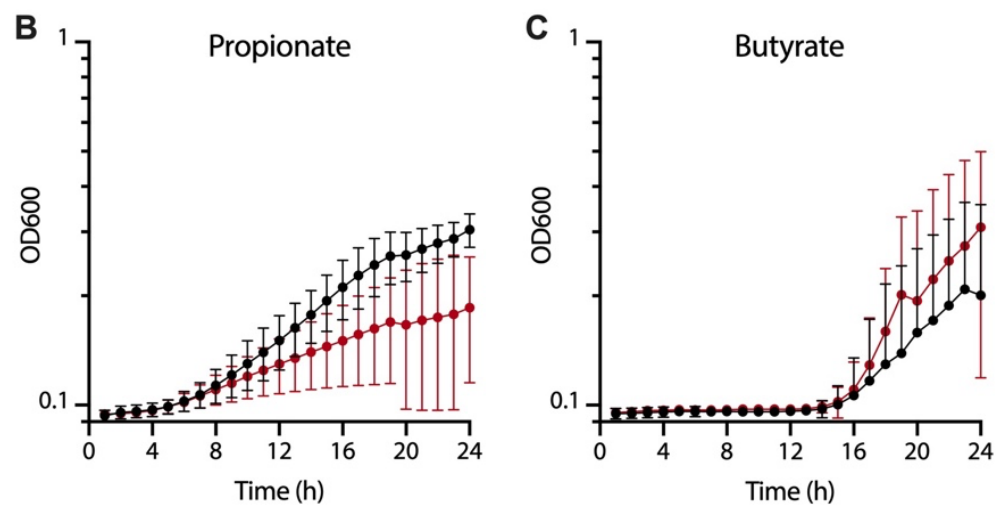

Figure 5. The $\Delta f a d X$ mutant is more susceptible to growth inhibition by propionate than wild type. Combined growth curves $(n=4)$ of wild type USA300 or the $\Delta$ fadX mutant in $100 \mathrm{mM}$ of the sodium salts of A) acetate, B) propionate, or $\mathbf{C}$ ) butyrate. Data shown are the mean $+/$ - standard deviation of three biological replicates.

\section{codY mutants are resistant to butyrate.}

Although occasional growth was detected in LB supplemented with butyrate after $\sim 15$ hours of incubation, it consistently occurred in only one of three technical replicates of a given sample (either wild type or $\Delta f a d X)$. These wells were plated onto LB and mannitol salt agar to confirm the absence of contamination and that the observed turbidity was due solely to $S$. aureus growth. To further investigate this phenomenon, we grew the $\Delta f a d X$ mutant for $24 \mathrm{~h}$ in LB, then plated ten-fold serial dilutions onto LB agar $+200 \mathrm{mM}$ sodium butyrate. Large colonies were occasionally observed in the $10^{-2}$ dilutions after 
368 colonies onto the same medium confirmed their resistance phenotype. We interpret these data to mean 369 that butyrate resistant mutants arose spontaneously in LB starter cultures, and that the occasional 370 turbidity in LB + butyrate cultures after $\sim 15$ hours represents growth after an extended lag phase resulting 371 from their extremely low starting abundance.

372 Growth curves in each SCFA were then performed to determine if the large colonies had a growth 373 advantage over the parental strain. We found that all four large colonies grew significantly faster in the 374 presence of butyrate than the parental strain, yet there were no differences in media supplemented with 375 acetate or propionate (Fig. 6A-C). We then sequenced their genomes to identify genetic determinant(s) 376 of butyrate resistance, and found two independent mutations in the gene encoding the GTP- and 377 branched chain amino acid-sensing global regulator CodY. The first mutation resulted in a premature 378 stop codon truncating the protein after 65 amino acids, while the second led to a 20 amino acid deletion 379 from a conserved region of the protein at codons 171-190 (Fig. 6D). We repeated growth curves using a 380 JE2 $\operatorname{cod} Y:$ :tn mutant (with an intact fadX gene) and confirmed that $\operatorname{cod} Y$ mutation alone was sufficient to 381 rescue growth in the presence of butyrate (Fig. 7A). Finally, we performed qRT-PCR on JE2 and the $382 \operatorname{cod} Y::$ th mutant to assay for fad operon expression and found that while the operon was modestly 383 induced in the mutant, none of the genes reached significance (Fig. 7B). These data, coupled with the 384 fact that codY mutants have similar levels of growth impairment in propionate is further evidence that 385 propionate and butyrate may act on different targets to inhibit $S$. aureus growth. 

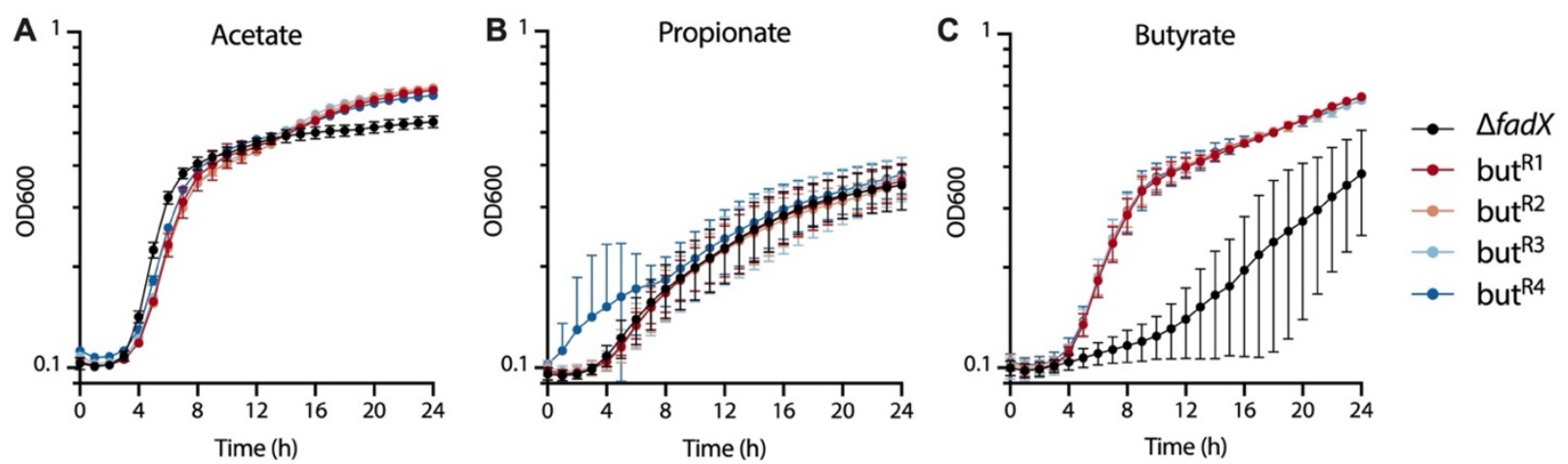

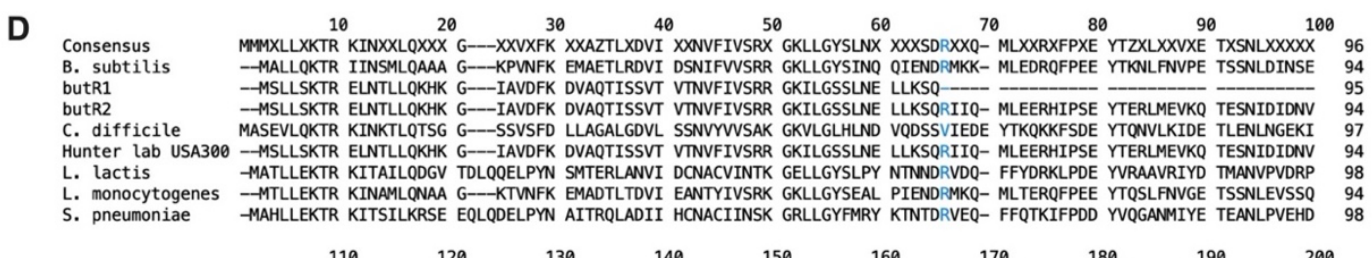

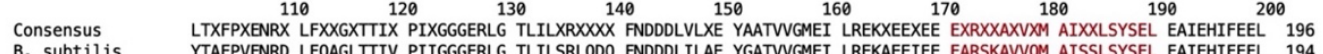
B. subtilis YTAFPVENRD LFQAGLTTIV PIIGGGERLG TLILSRLQDQ FNDDDLILAE YGATVVGMEI LREKAEEIEE EARSKAVVOM AISSLSYSEL EAIEHIFEEL 194

\begin{tabular}{llll}
$\begin{array}{l}\text { butR1 } \\
\text { butR2 }\end{array}$ LTVFPPENRE LFIDSRTTIF PILGGGERLG TLVLGVHDD FNENDLVLGE YAATVIGMEI LREKHSEVEK & 195 \\
\hline
\end{tabular}

c. difficile LEIFPEEHGR L--OKYTTVV PILGSGRLG TLVLSRYSNS FNDDDLVIAE YSATVVGLEI LRAIGEELEE EMRKKAVVOM AIGTLSYSEL EAVEHIFAEL 195

Hunter lab USA300 LTVFPPENRE LFIDSRTTIF PILGGGERLG TLVLGRVHDD FNENDLVLGE YAATVIGMEI LREKHSEVEK EARDKAAITM AINSLSYSEK EAIEHIFEEL 194

L. lactis 19 LAIFPEESLG DFPKGVTTLA PIYGSGMRLG TFIMWREDGE FTDDDLVLVE LATTVIGVQL SNLKLEOMEE NIRKDTMATM AVNTLSYSEM KAVKAIIEEL 198

$\begin{array}{lll}\text { L. monocytogenes } & \text { YTAFPIENSE LFTKGLTTIV PIVGGERLG TLILSRLESN FTDDDLLLAE YGGTVVGMEI LHEKAEEIEE EARSRAVVOM AISSLSYSEL EAIEHIFDEL 194 } \\ \text { S. pneumoniae } & \text { MSIFPVESR DFPDGLTIIA PIHVSGIRLG SLIIWRNDKK FEDEDLVLVE IASTVVGIQL LNFQREEDEK NIRRRTAVTM AVNTLSYSEL RAVSAILGEL 198 }\end{array}$

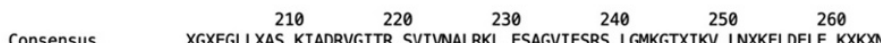

Consensus $\quad$ XGXEGLLXAS KIADRVGITR SVIVNALRKL ESAGVIESRS LGMKGTXIKV LNXKFLDELE KXKXNO

butR1

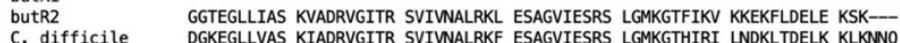

Hunter lab USA300 GGTEGLLIAS KVADRVGITR SVIVNALRKL ESAGVIESRS LGMKGTFIKV KKEKFLDELE KSK--

L. lactis DGEEGHVIAS VIADKIGITR SVIVNALRKL ESAGVIESRS LGMKGTYLKV LNTGLFDKLA GRNF--

L. monocytogenes NGKEGLLVAS KIADRVGITR SVIVNALRKL ESAGVIDSRS LGMKGTFIRV LNDKFLVELE KLKNN-
S. pneumoniae

Figure 6. Spontaneous $S$. aureus codY mutants are not inhibited by butyrate. Combined growth curves of the $\Delta \mathrm{fad} X$ mutant and butyrate-resistant derivatives in $100 \mathrm{mM} \mathrm{A}$ ) sodium acetate, B) sodium propionate, or C) sodium butyrate. D) Alignment of CodY protein sequences from diverse Gram-positive bacteria and $S$. aureus, including butyrate resistant mutants (but ${ }^{\mathrm{R} 1}$ and but ${ }^{\mathrm{R} 2}$ ). but ${ }^{\mathrm{R} 1}$ encodes a premature stop codon at position 66 (blue), while but ${ }^{\mathrm{R} 2-4}$ mutants have a 20 amino acid deletion from positions $171-190$ (red). but ${ }^{\mathrm{R} 3}$ and but ${ }^{\mathrm{R} 4}$ mutants were omitted from the alignment as their codY mutations are identical to but ${ }^{\mathrm{R} 2}$.
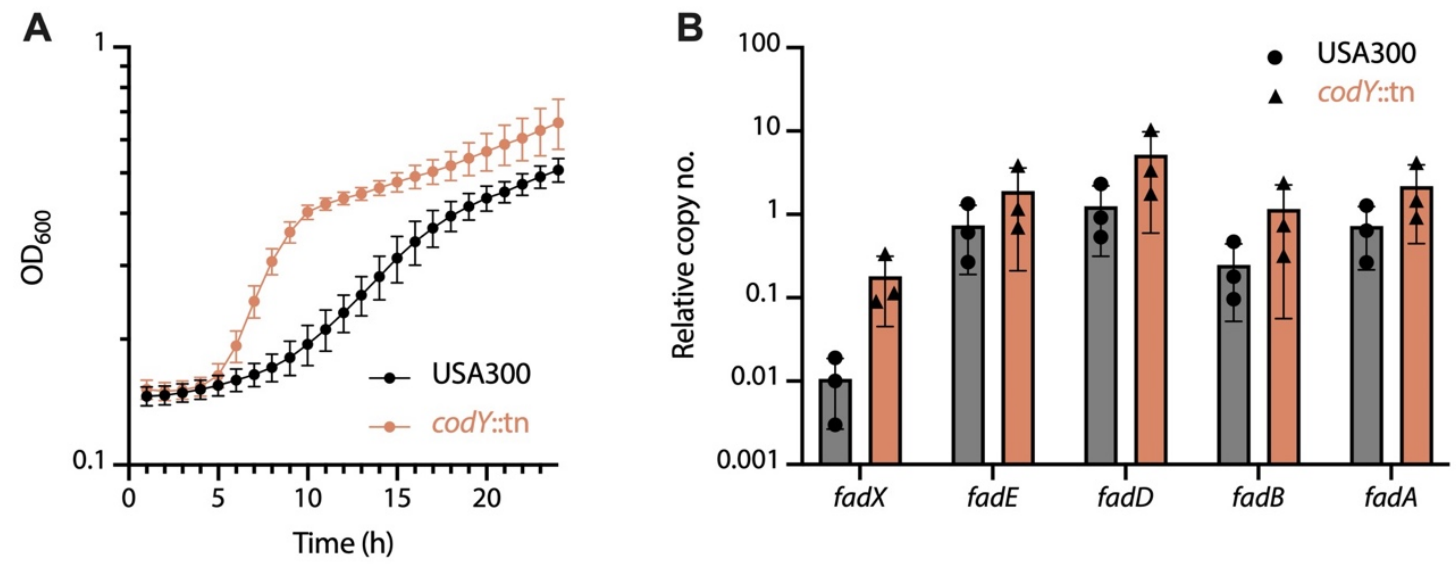

Figure 7. $\operatorname{cod} Y$ mutation is sufficient to escape butyrate growth inhibition, though not likely through Fad activity. A) Growth of wild type S. aureus and a codY::tn mutant in $100 \mathrm{mM}$ sodium butyrate. B) Expression of the fad operon from the same strains as in A, grown in LB to an $\mathrm{OD}_{600}$ of approximately $0.2-0.3$. 
DISCUSSION

399 Although not lethal, CRS remains a significant source of morbidity for a large percentage of the population $400(\sim 15 \%)$, and recalcitrance to antibiotic therapy often requires invasive surgical intervention ${ }^{5}$. The recent 401 advances in bacterial community profiling by $16 S$ amplicon sequencing has revealed extensive 402 colonization of CRS sinus mucus with oral anaerobes and other taxa not frequently observed via 403 traditional culture-based methods ${ }^{4,6}$. While $S$. aureus is appreciated as a significant CRS pathogen, it 404 coexists with these communities and must adapt to the sinonasal microenvironment shaped by both host 405 and microbial processes. Many of the anaerobes associated with CRS release nutrients in the form sialic 406 acid and other carbohydrates, peptides and amino acids, and byproducts of mixed-acid fermentation ${ }^{6}$. 407 One such class of metabolites, short-chain fatty acids, are derived from amino acid-fermenting 408 anaerobes, particularly members of the Fusobacterium genus ${ }^{29}$. Our goals in this work were to (i) 409 determine if Fusobacterium nucleatum, a model member of the Fusobacteria, could impair S. aureus 410 growth, (ii) characterize the response of $S$. aureus to individual SCFAs, and (iii) identify potential 411 mechanisms of SCFA stress. We show that $F$. nucleatum produces millimolar amounts of the SCFAs acetate, propionate, and 413 butyrate, and that $S$. aureus has an extended lag phase and significant alterations to its transcriptome 414 when grown in F. nucleatum supernatants or control medium supplemented with SCFAs. Consistent with 415 a prior study, propionate and butyrate were both inhibitory to $S$. aureus growth, though we found that 416 butyrate was more potent in this regard ${ }^{26}$. Both SCFAs were sufficient to reduce expression of the master 417 regulator of virulence, agrA, and alter expression of metabolic pathways (cidA and the fad operon), in 418 support of the hypothesis that SCFAs were responsible for altered gene expression and the delayed lag 419 phase in F. nucleatum supernatants. Reduced agr-regulated virulence factor output may alter the 420 inflammatory tone of the CRS sinus environment, with the host response instead being directed towards 421 members of the anaerobic community rather than $S$. aureus. In support of this idea, CRS patients have 422 been reported to have circulating antibodies targeting Fusobacterium and Prevotella, members of which 423 were enriched in CRS sinus mucus in our previous study, and that they show a decline in these antibodies 424 after successful antibiotic therapy ${ }^{6,30}$. Alternatively, butyrate produced by anaerobes in the CRS sinus 
425 environment may occasionally select for $S$. aureus codY mutants, whose growth inhibition would be 426 relieved ${ }^{31}$. Such mutants overproduce numerous virulence factors, and as such, may exacerbate the 427 inflammatory response ${ }^{32}$. Whatever the case, the recent development of robust animal models of CRS 428 will facilitate our ability to test these hypotheses in vivo ${ }^{33,34}$.

While the mechanisms of action of propionate and butyrate on $S$. aureus are still unclear, our data

430 suggest that they may act on different targets. Propionate induced expression of the fad operon and a

431 fadX mutant exhibited worse growth in its presence than did the wild type strain. The fad operon 432 annotation suggests a role in fatty acid degradation, though it is unlikely that it acts directly on propionate, 433 as S. aureus showed no evidence of metabolizing it over time (Fig. 1C). Another possibility is that 434 propionate induces lipid membrane stress, and the Fad proteins may act to degrade or repair damaged 435 lipid species. This consistent with recent findings from human gut commensal Bacteroides, where 436 butyrate (rather than propionate) induced membrane stress in a species- and context-dependent 437 manner ${ }^{35}$. Butyryl-CoA levels were increased by Acyl-CoA enzymatic activity, suggesting that other CoAregulated enzymes could be starved of an essential cofactor, likely imparing several metabolic processes.

439 While butyrate also induced expression of fadX, we did not detect a mutant phenotype in LB 440 supplemented with $100 \mathrm{mM}$ butyrate, suggesting that the other members of the fad operon may 441 compensate for the loss of fadX under these conditions, or that they are less important for the response 442 to butyrate. Interestingly, we readily obtained spontaneous butyrate resistance in the form of codY 443 mutations, while we did not for propionate. Further, butyrate resistant $\operatorname{cod} Y$ mutants were as sensitive to 444 propionate as the parent strain, suggesting that each SCFA may have unique mechanisms of action on 445 S. aureus. CodY is an allosteric transcriptional regulator whose affinity for specific motifs in promoter 446 regions is dictated by the levels of GTP and branched chain amino acids in the cell ${ }^{36}$. As it regulates 447 hundreds of genes in $S$. aureus, determining which CodY target(s) are responsible for bypassing 448 butyrate-mediated growth inhibition are beyond the scope of this work, though transposon screens in a $449 \operatorname{cod} Y$ mutant background may prove fruitful in this regard.

450 In summary, we have identified a possible mechanism by which anaerobic bacteria in 451 polymicrobial airway infections may influence $S$. aureus growth and physiology via the activity of the 
452 short-chain fatty acids propionate and butyrate, and have identified the fad operon and the CodY regulon 453 as possible mechanisms of resistance, respectively. Our study has some limitations, as the experiments 454 were performed in F. nucleatum supernatants in vitro and in defined media conditions rather than in the 455 context of intact anaerobic communities or two-species co-cultures. Additionally, our experiments lack 456 the potential contributions of the host, such as reactive oxygen and nitrogen species, or cationic 457 antimicrobial peptides ${ }^{37,38}$. Despite these limitations, the genetic approach taken here is informative and 458 amenable to translation into animal models for further dissection of the effects of CRS bacterial 459 communities on S. aureus pathogenesis.

\section{ACKNOWLEDGEMENTS}

462 We acknowledge the UMN Genomics Center and Paige Marsolek for NanoString assistance, and 463 members of the Hunter laboratory for critical feedback on the manuscript. This work was supported by a 464 National Institute of Dental and Craniofacial T32 Fellowship (\#T90DE0227232) awarded to JRF, a 465 National Heart, Lung, and Blood Institute Research Project Grant (1R01HL136919) to RCH, and an 466 Administrative Research Supplement (HL136919-03S1) to ARV. The funders had no role in study design, 467 data collection and interpretation, or the decision to submit the work for publication.

\section{REFERENCES}

470 1. Hastan, D. et al. Chronic rhinosinusitis in Europe--an underestimated disease. A GA ${ }^{2}$ LEN study. $471 \quad$ Allergy 66, 1216-1223 (2011).

472 2. Orlandi, R. R. et al. International Consensus Statement on Allergy and Rhinology: Rhinosinusitis. 473 Int Forum Allergy Rhinol 6 Suppl 1, S22-209 (2016).

474 3. Tu, Y. et al. Mucus composition abnormalities in sinonasal mucosa of chronic rhinosinusitis with 475 and without nasal polyps. Inflammation (2021) doi:10.1007/s10753-021-01471-6.

476 4. Hoggard, M. et al. Evidence of microbiota dysbiosis in chronic rhinosinusitis. Int Forum Allergy $477 \quad$ Rhinol 7, 230-239 (2017). 
478 5. Hoggard, M. et al. Chronic Rhinosinusitis and the Evolving Understanding of Microbial Ecology in 479 Chronic Inflammatory Mucosal Disease. Clin Microbiol Rev 30, 321-348 (2017).

480 6. Lucas, S. K. et al. Anaerobic microbiota derived from the upper airways impact Staphylococcus 481 aureus physiology. Infect Immun IAI0015321 (2021) doi:10.1128/IAI.00153-21.

482 7. Bhattacharyya, N. Incremental health care utilization and expenditures for chronic rhinosinusitis in 483 the United States. Ann Otol Rhinol Laryngol 120, 423-427 (2011).

484 8. Bhattacharyya, N. \& Kepnes, L. J. Assessment of trends in antimicrobial resistance in chronic 485 rhinosinusitis. Ann Otol Rhinol Laryngol 117, 448-452 (2008).

486 9. Feazel, L. M., Robertson, C. E., Ramakrishnan, V. R. \& Frank, D. N. Microbiome complexity and 487 Staphylococcus aureus in chronic rhinosinusitis. Laryngoscope 122, 467-472 (2012).

488 10. Ramakrishnan, V. R., Feazel, L. M., Abrass, L. J. \& Frank, D. N. Prevalence and abundance of 489 Staphylococcus aureus in the middle meatus of patients with chronic rhinosinusitis, nasal polyps, 490 and asthma. Int Forum Allergy Rhinol 3, 267-271 (2013).

491 11. Bardy, J. J. et al. Staphylococcus aureus from patients with chronic rhinosinusitis show minimal 492 genetic association between polyp and non-polyp phenotypes. BMC Ear Nose Throat Disord 18, 16 493 (2018).

494 12. Wagner Mackenzie, B. et al. Bacterial community collapse: a meta-analysis of the sinonasal 495 microbiota in chronic rhinosinusitis. Environ Microbiol 19, 381-392 (2017).

496 13. Kapatral, V. et al. Genome sequence and analysis of the oral bacterium Fusobacterium nucleatum 497 strain ATCC 25586. J Bacteriol 184, 2005-2018 (2002).

498 14. Mirković, B. et al. The Role of Short-Chain Fatty Acids, Produced by Anaerobic Bacteria, in the 499 Cystic Fibrosis Airway. Am J Respir Crit Care Med 192, 1314-1324 (2015).

500 15. Thurlow, L. R., Joshi, G. S. \& Richardson, A. R. Virulence strategies of the dominant USA300 501 lineage of community-associated methicillin-resistant Staphylococcus aureus (CA-MRSA). FEMS $502 \quad$ Immunol Med Microbiol 65, 5-22 (2012).

503 16. Merritt, J. H., Kadouri, D. E. \& O'Toole, G. A. Growing and analyzing static biofilms. Curr Protoc $504 \quad$ Microbiol Chapter 1, Unit 1B.1 (2005). 
505 17. Kolde, Raivo. pheatmap.

506 18. Monk, I. R., Shah, I. M., Xu, M., Tan, M.-W. \& Foster, T. J. Transforming the untransformable:

507 application of direct transformation to manipulate genetically Staphylococcus aureus and

508 Staphylococcus epidermidis. mBio 3, (2012).

509 19. De Baere, S. et al. Development of a HPLC-UV method for the quantitative determination of four 510 short-chain fatty acids and lactic acid produced by intestinal bacteria during in vitro fermentation. $J$ $511 \quad$ Pharm Biomed Anal 80, 107-115 (2013).

512 20. Zilm, P. S., Gully, N. J. \& Rogers, A. H. Growth pH and transient increases in amino acid availability 513 influence polyglucose synthesis by Fusobacterium nucleatum grown in continuous culture. FEMS $514 \quad$ Microbiol Lett 215, 203-208 (2002).

515 21. Sadykov, M. R. et al. Inactivation of the Pta-AckA pathway causes cell death in Staphylococcus $516 \quad$ aureus. J Bacteriol 195, 3035-3044 (2013).

517 22. Janzon, L. \& Arvidson, S. The role of the delta-lysin gene $(h / d)$ in the regulation of virulence genes 518 by the accessory gene regulator (agr) in Staphylococcus aureus. EMBO J 9, 1391-1399 (1990).

519 23. Olson, M. E., King, J. M., Yahr, T. L. \& Horswill, A. R. Sialic acid catabolism in Staphylococcus aureus. J Bacteriol 195, 1779-1788 (2013).

24. Agarwal, K. et al. Glycan cross-feeding supports mutualism between Fusobacterium and the

25. Pohl, K. et al. CodY in Staphylococcus aureus: a regulatory link between metabolism and virulence gene expression. J Bacteriol 191, 2953-2963 (2009).

26. Jeong, S., Kim, H. Y., Kim, A. R., Yun, C.-H. \& Han, S. H. Propionate Ameliorates Staphylococcus aureus Skin Infection by Attenuating Bacterial Growth. Front Microbiol 10, 1363 (2019).

27. Saravia-Otten, P., Müller, H. P. \& Arvidson, S. Transcription of Staphylococcus aureus fibronectin

28. Ranjit, D. K., Endres, J. L. \& Bayles, K. W. Staphylococcus aureus CidA and LrgA proteins exhibit 
29. Rogers, A. H., Zilm, P. S., Gully, N. J., Pfennig, A. L. \& Marsh, P. D. Aspects of the growth and metabolism of Fusobacterium nucleatum ATCC 10953 in continuous culture. Oral Microbiol Immunol 6, 250-255 (1991).

30. Brook, I. \& Yocum, P. Immune response to Fusobacterium nucleatum and Prevotella intermedia in patients with chronic maxillary sinusitis. Ann Otol Rhinol Laryngol 108, 293-295 (1999).

31. Cho, D.-Y. et al. Contribution of Short Chain Fatty Acids to the Growth of Pseudomonas aeruginosa in Rhinosinusitis. Front. Cell. Infect. Microbiol. 10, 412 (2020).

32. Majerczyk, C. D. et al. Staphylococcus aureus CodY negatively regulates virulence gene expression. J Bacteriol 190, 2257-2265 (2008).

33. Cho, D.-Y. et al. Sinus Microanatomy and Microbiota in a Rabbit Model of Rhinosinusitis. Front Cell

35. Park, S.-Y. et al. Strain-level fitness in the gut microbiome is an emergent property of glycans and a single metabolite. Cell 185, 513-529.e21 (2022).

547 36. Brinsmade, S. R. CodY, a master integrator of metabolism and virulence in Gram-positive bacteria. Curr Genet 63, 417-425 (2017).

549 37. Jardeleza, C. et al. Gene expression differences in nitric oxide and reactive oxygen species regulation point to an altered innate immune response in chronic rhinosinusitis. Int Forum Allergy 